\title{
Alzheimer's Disease and Non-Insulin-Dependent Diabetes Mellitus: Common Features Do Not Make Common Bedfellows
}

A Izheimer's Disease (AD) and non-insulin-dependent diabetes mellitus (NIDDM) are two of the most common and devastating health problems afflicting older adults. $\mathrm{AD}$ and NIDDM share a number of common features. Both conditions can have an enormous impact on a patient's quality of life, and both are associated with substantial health care costs. AD and NIDDM both have names that contain little useful information. $A D$ is named after an obscure pathologist primarily to avoid use of a term that would convey directly its devastating impact on cognitive functioning. NIDDM is a non name that obscures the reality that many patients with NIDDM are in fact treated with insulin. Furthermore, a substantial proportion of patients with NIDDM rarely or never have significant amounts of sugar in their urine, certainly not enough to give their urine the sweet taste which is the basis for the name diabetes mellitus.

Both AD and NIDDM are subjects of intensive investigation by the scientific community, and substantial resources of relevant NIH institutes are committed to support research about them. Perhaps my only tangible qualification to write about both disorders is that I serve on the advisory board of two NIH-supported Center grants at the University of Michigan: the Michigan Diabetes Research and Training Center and the Michigan Alzheimer's Disease Research Center. At this time, a major research emphasis both in NIDDM and in $A D$ is the search for genetic markers for these conditions, since both have a genetic predisposition. Specific genetic defects have now been identified in a small number of families with NIDDM ${ }^{1}$ and with AD. $^{2}$ The information obtained thus far suggests that there may be considerable heterogeneity in the genetic factors which contribute to both of these conditions.

The importance of genetic markers to advance understanding of these disorders relates, in part, to the difficulty in establishing the clinical phenotype for both NIDDM and AD. Thus, studies relating clinical phenotype to genotypic markers are particularly important. In this issue of the Journal of the American Geriatrics Society, Nielson et al. have used apolipoprotein-E genotyping to correlate with the clinical phenotype in 265 dementia patients participating in a University-based Alzheimer's Disease Research Center Clinic. ${ }^{3}$ Included in this population were 15 patients who also had diabetes mellitus by history. In confirmation of findings from other studies (reference 4 and others referenced by Nielson et al.), they found a high prevalence rate of apo-E4 and a low prevalence rate of apo-E2 in patients meeting criteria for possible or probable AD. Patients with other clinical types of dementia and those dementia patients with diabetes mellitus had apo-E genotype distribution similar to the general population.

\section{See also p 897}

The apoprotein-E genotype may play a pathophysiological role in the formation of the amyloid plaques that are characteristic of $A D .^{2,5}$ Some evidence suggests that apo-E may interact with the beta-amyloid protein, a secreted product of the amyloid precursor protein or APP, ${ }^{6}$ to influence its tertiary structure. ${ }^{7}$ The presence of apo-E4 may affect the tertiary structure of beta-amyloid protein to make it more likely to form non-soluble amyloid, thereby contributing to the formation of amyloid plaques, whereas the apo-E2 form may stabilize beta-amyloid protein and prevent formation of the non-soluble form of amyloid.

Here too there is a convergence between $A D$ and NIDDM. Deposition of amyloid deposits in pancreatic islet beta cells appears to be a consistent pathologic marker in NIDDM. An islet peptide known as amylin (also known as islet amyloid polypeptide or IAPP, not to be confused with APP!) has been identified as the key component of such amyloid deposits. ${ }^{8}$ Amylin is colocalized and cosecreted with insulin, but its physiologic role is unknown. ${ }^{9}$ Although amylin and beta amyloid protein are both cell secretory products, and both have the propensity under some circumstances to form insoluble amyloid fibrils that are toxic to host cells, ${ }^{5,10}$ it should be emphasized that the polypeptides themselves are completely different. While the role of either of these molecules in the primary parhogenesis of AD or NIDDM is still unclear, it is tempting to speculate that an alteration in protein folding ${ }^{11}$ leading to aggregation and amyloid deposits may be a key mechanism in both diseases.

Because of these common features of AD and NIDDM, one might postulate that it would be common to find both conditions in given susceptible individuals. However the opposite may be true. Nielson et al. found that only one of the 15 people with a diagnosis of diabetes in their dementia population met criteria for $\mathrm{AD}$, representing only $0.8 \%$ of the $\mathrm{AD}$ group, perhaps $10 \%$ or less of the expected prevalence rate of NIDDM in an older population. ${ }^{12}$ This finding agrees with other reports suggesting a low prevalence rate of NIDDM among patients with AD. What is the explanation for this finding?

As Nielson et al. point out in their paper, there are a number of factors in their study and others in this area that limit the ability to draw firm conclusions about the relationship, or lack thereof, between a diagnosis of $\mathrm{AD}$ and 
NIDDM. Since none of the studies to date are population based, patient selection can be a confounding factor. It is conceivable that patients with NIDDM who also have dementia are less likely to end up in a research oriented clinic for cognitive disorders than patients with dementia who do not have diabetes mellitus. A more serious concern is the problem of phenotype characterization. Clinical criteria for AD virtually exclude anyone with identifiable cerebrovascular disease. Given the high prevalence of evidence of cerebrovascular disease among older patients with NIDDM, many NIDDM patients are automatically excluded from the AD category. This is where the genotyping carried out by Nielson et al. may be helpful. If a high percentage of the NIDDM patients in their sample indeed had unrecognized $A D$, one might have expected a higher prevalence of the apo-E4 genotype. There is also a major phenotype problem regarding the diagnosis of NIDDM. Population-based studies in the United States suggest that up to $50 \%$ of patients who meet current diagnostic criteria for NIDDM have not been diagnosed. ${ }^{13}$ Thus, the true prevalence of NIDDM among the AD patients in the paper by Nielson et al. cannot be established in the absence of specific testing of glucose tolerance status. The diagnosis of diabetes in this study was based entirely on clinical history. Only a minority of the patients had even a fasting serum glucose level tested.

More intriguing would be the possibility that some aspect of AD prevents NIDDM and/or some aspect of NIDDM protects against $A D$. A simple hypothesis is that the weight loss that often accompanies $\mathrm{AD}^{14}$ leads to a reduction in obesity-related insulin resistance, thereby tending to prevent the development of hyperglycemia. Could there be a positive benefit of chronic hyperglycemia to reduce the rate of development of degenerative pathologic lesions in the brain by simply enhancing access of brain tissue to an important fuel? There is evidence that reduced neuronal oxidative metabolism, a possible consequence of low fuel availability, can result in a change in APP processing that increases the likelihood that beta-amyloid peptide will be produced. ${ }^{15}$ Could adaptive mechanisms in response to one disorder of protein folding reduce the likelihood of development of a second such disorder? For example, if there are generalized antiprotein aggregation biochemical responses to the formation of aggregated protein resulting from a genetic defect of overproduction of an amyloid precursor protein, such mechanisms might reduce the likelihood of accumulation of protein aggregates in other tissues. Clearly, additional work is needed to first determine whether the presence of AD or NIDDM actually reduces risk for the other condition, and, if so, what mechanisms might contribute to such protection.

Jeffrey B. Halter, $M D$ University of Michigan Medical School and Geriatric Research Education and Clinical Center Veterans Affairs Medical Center, Ann Arbor, MI

\section{REFERENCES}

1. Turner RC, Hattersley AT, Shaw JTE et al. Type II diabetes: Clinical aspects of molecular biological studies. Diabetes 1995;44:1-10.

2. Hyman BT, Tanzi R. Molecular epidemiolegy of Alzheimer's Diseasc. N Engl J Med 199.5;333:1283-1284.

3. Niclson KA, Nolan JH, Berchtold NC et al. Apolipoprotein-E genotyping of diabetic dementia patients: Is diabetes rare in Alzheimer's Disease? J Am Geriatr Soc 1996;44:0000-0000.

4. Polvikoski T, Sulkava R, Haltia $M$ tt al. Apolipoprotein E, dementia, and cortical deposition of $\beta$-amyloid protein. N Engl J Med 1995;33.3:12421247.

5. Yankner BA, Mesulam MM. $\beta$-amyloid and the pathogenesis of Alzheimer's Disease. N Engl J Med 1991;325:1849-1857.

6. Busciglio J, Gabuzda DH, Matsudaira P et al. Generation of beta-amyloid in the secretory pathway in neuronal and nonneuronal cells. Proc Natl Acad Sc USA 1993;90:2092-2096.

7. Strittmatter WJ, Roses AD. Apolipoprotcin E and Alxheimer disease. Proc Natl Acad Sci USA 199.5;92:4725-4727.

8. Johnson $\mathrm{KH}$, O'Brien TI), Betsholtz. $\mathrm{C}$ et al. Islet amyloid, islet-amyloid polypeptide, and diabetes mellitus. N Engl J Med 1989;321:513-518.

9. Edwards BJA, Morley JE. Minireview: Amylin. Life Sci 1992;51:1899-1912.

10. Lorenzo A, Razzaboni B, Weir GC et al. Pancreatic islet cell toxicity of amylin associated with type-2 diaberes mellitus. Nature 1994;368:756-760.

11. Taubes G. Misfolding the way to disease. Science 1996;271:1493-1495.

12. Cieiss LS, Herman WH, Goldschmid MG et al. Surveillance for diabetes mellitus: United Statcs, 1980-1989. MMWR C.DC Surveillance Summaries. 1993;42:1-20.

13. Harris MI. Undiagnosed NIDDM: Clinical and public health issues. Diabetes Care 1993;16:642-652.

14. Poehlman ET. Regulation of energy expenditure in aging humans. J Am Geriatr Soc 1993;41:552-559.

15. Gabuzda D, Busciglio J, Chen LB et al. Inhibition of energy metabolism alters the processing of amyloid precursor protein and induces a potentially amyloidogenic derivative. J Biol Chem 1994;269:13623-13628. 